Citation: M. Grasso, F. Randelli (2021). Le responsabilità e il ruolo degli agenti di destabilizzazione nella transizione verso la sostenibilità: un framework teorico. Bollettino della Società Geografica Italiana serie 14, 4(2): 33-44. doi: $10.36253 /$ bsgi-1135

Copyright:@2021 M. Grasso, F. Randelli. This is an open access, peer-reviewed article published by Firenze University Press (http://www.fupress.com/bsgi) and distributed under the terms of the Creative Commons Attribution License, which permits unrestricted use, distribution, and reproduction in any medium, provided the original author and source are credited.

Data Availability Statement: All relevant data are within the paper and its Supporting Information files.

Competing Interests: The Author(s) declare(s) no conflict of interest.

For Italian evaluation purposes, Marco Grasso takes responsibility for sections 2 and 4, Filippo Randelli for sections 3 and 5 , the two authors jointly for sections 1 and 6.

\section{Le responsabilità e il ruolo degli agenti di destabilizzazione nella transizione verso la sostenibilità: un framework teorico}

\author{
The responsibilities and role of destabilizing agents in the \\ transition towards sustainability: a theoretical framework
}

\author{
Marco Grasso $^{1}$, Filippo Randelli ${ }^{2}$ \\ ${ }^{1}$ Dipartimento di Sociologia e Ricerca Sociale, Università degli Studi di Milano-Bicocca, \\ Italia \\ ${ }^{2}$ Dipartimento di Scienze per l'Economia e l'Impresa, Università degli Studi di Firenze, \\ Italia \\ E-mail: marco.grasso@unimib.it; filippo.randelli@unifi.it
}

\begin{abstract}
Since it is not beneficial to everyone, the sustainability transition is a complex process and many mechanisms hinder its evolution. Some agents involved in this transition shoulder significant costs and burdens: even if some agents can shoulder part of them, it is unlikely that all agents will spontaneously comply with the responsibility that the sustainability transition imposes on them. This paper intends to investigate how, in the context of the sustainability transition, the relevant agents must/can comply with the duties demanded by this responsibility (which can be defined as "first order" responsibility). Indeed, some agents do not comply with their own (first order) responsibility and with the consequent obligations. To induce such (first order) agents to act in accordance with their (first order) responsibility, the notion of "second order" responsibility - that is, the responsibility that other agents ensure that the first order agents act on the basis of their (first order) responsibility - should be employed. In this normative perspective, the work proposes a theoretical-analytical framework within which second order agents - which in a pragmatic perspective can be defined as "agents of destabilization" (divided into primary and operational) - take action to win the resistance of first order agents in order to favor the sustainability transition.
\end{abstract}

Keywords: sustainability transition, responsibility, agents of destabilization.

Riassunto. La transizione verso la sostenibilità è complessa poiché molti meccanismi ne ostacolano l'evoluzione in quanto è un processo non vantaggioso per tutti. Alcuni agenti coinvolti in tale transizione devono sostenere costi e oneri rilevanti: anche se alcuni agenti possono sostenerne una parte, è dubbio che essi si conformino spontaneamente alle responsabilità che la transizione verso la sostenibilità impone loro. Il presente lavoro intende indagare come, nel contesto della transizione verso la sostenibilità, gli agenti rilevanti debbano/possano ottemperare gli obblighi imposti da tale responsabilità - definita di primo livello. Alcuni agenti, infatti, non si conformano alla propria responsabilità (di primo livello) e ai conseguenti obblighi verso la sostenibilità. Per indurre tali agenti ad agire secondo quanto dettato dalla loro responsabilità (di primo livello) è 
necessario analizzare la responsabilità di secondo livello - cioè, la responsabilità che altri agenti (di secondo livello) assicurino che gli agenti (di primo livello) si impegnino verso la sostenibilità, come richiesto dalle loro responsabilità (di primo livello). In questa prospettiva normativa, il lavoro propone un framework teorico-analitico entro cui tali agenti di secondo livello - che in ottica pragmatica possono essere definiti "agenti di destabilizzazione" (primari e operativi) - si attivano per contribuire a vincere il potere e la resistenza degli agenti di primo livello con l'obiettivo di favorire la transizione verso la sostenibilità

Parole chiave: transizione verso la sostenibilità, responsabilità, agenti di destabilizzazione.

\section{Introduzione}

Nell'agosto del 2021 il rapporto dell'Intergovernmental Panel on Climate Change (IPCC) delle Nazioni Unite, un codice rosso per l'umanità, ha indicato che se si vogliono evitare conseguenze ambientali catastrofiche, tra cui la grave perdita di biodiversità, la compromissione irreparabile delle barriere coralline e una significativa riduzione delle comunità insulari, sono da subito necessarie profonde trasformazioni nei nostri sistemi socioeconomici (IPCC 2021). Nell'aprile 2019, le Nazioni Unite hanno pubblicato il rapporto più completo sullo stato della biodiversità e dei servizi ecosistemici (IPBES 2019) a partire dal fondamentale Millenium Ecosystem Assessment (2005). I risultati sono drammatici: due specie di anfibi su cinque sono a rischio di estinzione, come un terzo delle barriere coralline e quasi un terzo delle altre specie marine. Le profonde mutazioni e le catastrofi ambientali avranno pesanti costi economici: soltanto nel settore agricolo la perdita di impollinatori ha messo a rischio 577 miliardi di dollari di produzione, mentre il degrado del suolo ha ridotto la produttività agricola globale del $23 \%$ (Holt-Giménez, Altieri 2013). La questione della necessità di radicali trasformazioni nel nostro sistema socio-economico è diventata impellente e nel prossimo futuro sarà prioritaria nei dibattiti politici e accademici (Hölscher et al. 2018; Schot, Kanger 2018; Kivimaa et al. 2021).

Tuttavia non è sempre chiaro chi debba ritenersi responsabile per la transizione verso la sostenibilità. Una prima tipologia di risposta, del tutto plausibile in un regime economico dominato dal pensiero e dalle politiche capitaliste-liberiste, individua nelle imprese i soggetti che favoriscono l'introduzione di nuove tecnologie e processi produttivi progressivamente più rispettosi dell'ambiente. Ma perché, per esempio, un'impresa automobilistica che produce e vende sul mercato con discreti profitti (FCA, Renault, Toyota, etc...) dovrebbe modifi- care e/o interrompere il proprio core business per cercare nuove strade verso la transizione, inizialmente incerte e senza dubbio costose e poco profittevoli? E ancora, perché un'impresa operante nel settore Oil \& Gas (Eni, BP, ExxonMobil, Saudi Aramco, etc...) dovrebbe abbandonare i combustibili fossili e abbracciare la transizione verso le energie rinnovabili? Attualmente, nella migliore delle ipotesi, le imprese esistenti (incumbents) introducono delle innovazioni incrementali, ma non sembrano avere reale interesse a cambiare il regime socio-tecnologico in cui operano (Geels 2004). Ben diverse sono le motivazioni delle nuove imprese, o start-up, che fin dalla loro creazione introducono processi, tecnologie e comportamenti virtuosi nei confronti dell'ambiente. Tuttavia, ancora oggi la loro forza economica e politica, seppur in crescita, rimane limitata ed è difficile pensare alla loro decisa affermazione nel breve periodo. Quindi, sicuramente avranno un ruolo nella transizione verso la sostenibilità, ma non sembrano in grado di dettarne $\mathrm{i}$ tempi e i modi, che rimangono lenti e incerti.

Una seconda tipologia di risposta indica negli stati i responsabili della transizione. Questi possono introdurre cambiamenti radicali e finanziare nuovi investimenti, ma ciò avrebbe un impatto sulle finanze pubbliche verosimilmente dirompente. Alla luce dell'indebitamento pubblico dilagante e della crescente crisi di legittimità, risulta impensabile assegnare la responsabilità della transizione verso la sostenibilità esclusivamente agli stati, né tantomeno è plausibile pensare di scaricarla solo sulle regioni e sulle amministrazioni locali.

Una terza tipologia di risposta indica i consumatori, i quali attraverso le loro scelte possono agire come principali responsabili del cambiamento. Il ruolo dei consumatori è stato per troppo tempo ignorato (Grahber et al. 2008) ed è indubbio che negli ultimi anni la loro capacità di incidere sia cresciuta in modo significativo (Graziano, Forno 2012; Randelli, Rocchi 2017). Tuttavia, sarebbe quanto meno molto ottimistico e forse anche ingiusto, alla luce delle difficoltà economiche delle fasce deboli della società e più in generale in relazione alla mancanza di risorse finanziarie e non finanziarie dei consumatori, pensare che essi possano con le loro scelte farsi carico di una rapida e profonda trasformazione del nostro sistema socio-economico. Inoltre, i consumatori sono intrappolati entro modelli di consumo a elevata intensità fossile, dai quali è molto oneroso, in termini economici e personali, evadere (Grasso 2022).

Il quadro descritto è necessariamente affrettato e per ognuna delle opzioni riportate esiste una vasta letteratura sia a favore che critica. L'obiettivo di questo lavoro non è passare in rassegna i vari approcci alla responsabilità per la transizione verso la sostenibilità, quanto 
piuttosto gettare le basi per poter pervenire a una nuova visione d'insieme sulle diverse responsabilità per tale transizione. Il nostro punto di partenza quindi è la poca chiarezza sulle responsabilità che una profonda trasformazione dei nostri sistemi socio-economici richiede. Si discute molto su cosa sia necessario fare per salvare il nostro pianeta ma troppo poco e in modo piuttosto astratto su quali siano le responsabilità e su chi dovrebbero realisticamente gravare.

Il presente lavoro è strutturato come segue: la seconda sezione introduce il tema della responsabilità; la terza definisce la responsabilità di primo livello; la quarta la responsabilità di secondo livello; la quinta analizza e assegna le responsabilità di secondo livello a specifici "agenti di destabilizzazione" in un contesto come quello della transizione verso la sostenibilità.

\section{Responsabilità: elementi salienti per la transizione verso la sostenibilità}

A livello generale, le considerazioni di giustizia rappresentano, proteggono e promuovono le istanze dei soggetti coinvolti nella formulazione di modelli collaborativi accettabili e fattibili (Grasso 2007; Gardiner 2017). Soprattutto con riguardo alle questioni ambientali, la sola ricerca dell'efficienza economica non è sufficiente a mobilitare soggetti con interessi divergenti e a volte contrapposti, che oltretutto spesso sperimentano condizioni socio-economiche molto diverse. Le azioni collaborative hanno più probabilità di emergere quando il rischio è chiaro e immediato, quando la posta in gioco è relativamente bassa e quando gli incentivi al free-riding sono trascurabili. Sfortunatamente, non è questo il caso della transizione verso la sostenibilità. Di conseguenza, azioni collaborative in vista di tale transizione devono essere quanto più possibile condivise: una circostanza che è tanto più probabile quanto più tali iniziative sono accompagnate da considerazioni etiche e in particolare da obiettivi di giustizia. Una solida base etica, inoltre, conferisce alle azioni volte alla transizione maggiore legittimità attraverso cui persuadere agenti con interessi conflittuali a collaborare più proficuamente su azioni collettive mirate alla sostenibilità (Grasso, Markowitz 2015).

Il problema etico verosimilmente più complesso nell'approcciare la transizione verso la sostenibilità riguarda l'attribuzione delle responsabilità in modo che esse siano moralmente, legalmente, politicamente, socialmente e psicologicamente accettabili per i policymakers e per tutti gli stakeholders. La Convenzione Quadro sui Cambiamenti Climatici delle Nazioni Unite (UNFCCC), per esempio, si basa sul principio - diplo- maticamente efficace ma di scarsa applicabilità - delle "responsabilità comuni ma differenziate" per definire, anche se in modo piuttosto vago, i soggetti responsabili per i cambiamenti climatici.

Per muovere a un livello di analisi più efficace, è necessario chiarire che la responsabilità può essere (Shue 2017):

- Negativa o positiva: e richiedere agli agenti di astenersi dal compiere determinate azioni, o imporre agli agenti di agire in un certo modo.

- Speciale o generale: cioè, riguardare solo alcuni agenti (per esempio solo quelli coinvolti o solo quelli danneggiati), o concernere tutta l'umanità.

- Retrospettiva o prospettiva: la prima richiede a un agente di intraprendere un'azione sulla base di qualcosa che è accaduto nel passato, la seconda origina dal fatto che l'agente è nella posizione o ha la capacità di fare qualcosa nel futuro.

- Intenzionale o consequenziale: la prima, conosciuta anche come responsabilità morale, si basa sulla valutazione delle intenzioni, della volontà, del controllo, della conoscenza dell'agente; la seconda, spesso definita responsabilità causale, si limita a prendere in considerazione la "contribuzione causale" dell'agente a una circostanza.

- Individuale o collettiva: che cioè si focalizza solo sul ruolo dell'individuo rispetto ai problemi e alle soluzioni, o sul ruolo di gruppi formalizzati e stabili di individui (per esempio un'azienda, un partito politico, uno stato).

- Intragenerazionale o intergenerazionale: questa distinzione dipende da quanto gli obblighi richiesti dalla responsabilità siano protratti nel tempo. Nel caso della transizione verso la sostenibilità è chiaro che la dimensione intergenerazionale è fondamentale. Tuttavia è spesso complesso articolare e riconciliare principi etici che giustifichino adeguatamente la responsabilità su periodi temporale estesi.

E opportuno inoltre precisare che fino a ora sono state considerate forme di responsabilità di "primo livello" (che per semplicità vengono comunemente definite unicamente "responsabilità"), cioè di responsabilità che gli agenti hanno di fare o di non fare qualcosa (cioè di agire o di non agire in determinati modi). Maggiori dettagli sulla responsabilità di primo livello nel caso della transizione verso la sostenibilità sono forniti nel paragrafo successivo.

Una seconda cruciale dimensione riguarda le responsabilità di "secondo livello", cioè le responsabilità di assicurare che gli agenti che hanno responsabilità di primo livello agiscano coerentemente con esse (per esempio, le Agenzie Regionali per la Protezione Ambientale hanno la responsabilità - di secondo livello - di assicurare che le aziende inquinanti rispettino i limiti di 
emissione imposti loro sulla base della loro responsabilità - di primo livello). Nel caso della transizione verso la sostenibilità le responsabilità di secondo livello sono fondamentali: infatti, per costruire e utilizzare le capacità che permettono ai sistemi socio-economici di muovere verso di essa, il ruolo degli agenti con responsabilità di secondo livello - governi, organizzazioni internazionali, centri di ricerca, università, agenzie per l'innovazione, ma anche leader religiosi e spirituali, scrittori, artisti, individui carismatici - è imprescindibile. Questo articolo le analizza nel dettaglio in un successivo paragrafo.

Le diverse nozioni di responsabilità riportate non dovrebbero essere enfatizzate in quanto nella realtà spesso si sovrappongono (Grasso, Tàbara 2019); tuttavia esse sono di aiuto per esplorare il terreno - scivoloso - delle responsabilità per la transizione verso la sostenibilità.

Alla luce delle considerazioni condotte è possibile sviluppare un framework teorico, normativamente solido e quindi moralmente cogente per comprendere e assegnare le responsabilità in un contesto come quello della transizione verso la sostenibilità in cui manca un unico "centro", cioè dove le decisioni vengono prese a scale geografiche diverse da attori differenti, che spesso hanno limitata capacità e/o volontà di coordinare le proprie azioni. La definizione di tale framework è, come detto, l'obiettivo principale di questo articolo.

\section{Responsabilità di primo livello}

La responsabilità di primo livello può essere definita come la responsabilità di specifici agenti (individui, imprese, istituzioni) di "performing or omitting certain acts” (O’Neil 2001, 2005; Caney 2014). In altre parole, nel nostro contesto di riferimento, determinati agenti devono assumersi la responsabilità di modificare i propri comportamenti coerentemente con quanto richiesto dalla sostenibilità. I cambiamenti che derivano dalla assunzione di responsabilità sono indotti sia dai nuovi comportamenti meno impattanti sull'ambiente che dall'abbandono dei vecchi comportamenti ormai insostenibili. Per comportamenti si intendono le azioni poste in essere nell'atto di produrre, utilizzare o consumare.

La transizione verso la sostenibilità ha ormai una solida base teorica e metodologica che va individuata soprattutto nella "Geografia della Transizione" (GT) e nella rivista Environmental Innovation and Societal Transitions quale punto di riferimento scientifico. La maggior parte degli studi sulla GT si concentrano sull'emergere delle innovazioni ambientali (green innovations). La logica sottesa è che le innovazioni ambientali crescono e si diffondono grazie a processi di apprendi- mento e al miglioramento delle loro performances; i progressivi miglioramenti diminuiscono i costi di produzione che ne determinano la definitiva diffusione.

La transizione è un processo graduale che ha bisogno del supporto della politica, il cui obiettivo deve essere quello di incentivare la ricerca e la diffusione delle innovazioni, sopperendo inizialmente ai costi elevati - quindi a uno dei tipici market failures - con incentivi all'acquisto e agli investimenti (per esempio, feed in tariffs). Le innovazioni ambientali possono così crescere fino al punto di svolta (breaktrough) in cui diventano dominanti, rendendo obsolete le vecchie tecnologie e le pratiche non più sostenibili né profittevoli. Nello schema logico della GT si assumono quindi: i) la responsabilità di primo livello, ii) gli imprenditori pionieri (nuove imprese, start-ups) che introducono le innovazioni ambientali, iii) le istituzioni pubbliche che sostengono il processo con adeguate politiche e infine iv) le imprese esistenti (incumbents) che per rimanere competitive dovranno coevolvere adeguandosi ai nuovi standard.

Questa logica, seppur qui semplificata, è alla base delle due principali architetture concettuali della GT: la technological innovation systems (TIS) e la multi-level perspective (MLP). I due framework hanno avuto innumerevoli applicazioni in studi sulla transizione nei principali settori quali energia, trasporti, rifiuti, agricoltura, turismo, etc.

Il TIS framework è emerso agli inizi degli anni Novanta e fonda le sue radici nella letteratura dell'economia evoluzionista (Lundvall 1992; Nelson 1993). Un TIS può essere definito come "a dynamic network of agents interacting in a specific production under a particular institutional infrastructure and involved in the generation, diffusion and utilisation of a specific technology or product" (Carlsson Stankiewicz 1991, 111). La ricerca sui TIS ha trovato larga applicazione sullo studio della transizione verso la sostenibilità (per una rassegna si veda Markard et al. 2012). Un sistema innovativo è dunque un costrutto analitico, cioè uno strumento per meglio comprenderne le dinamiche evolutive (Bergek et al. 2008). Ne consegue che un TIS e la rete di agenti che lo compongono hanno un ciclo di vita che inizia con la fase di formazione e termina con la definitiva crescita e affermazione dell'innovazione ambientale (Markard Truffer, 2008). L'obiettivo degli studi sui TIS è quello di evidenziare punti di forza e di debolezza del processo di affermazione dell'innovazione ambientale al fine di indicare le misure più adatte ai policy maker. Generalmente, sono stati identificati diversi processi o funzioni chiave (si veda per esempio Bergek et al. 2008) che caratterizzano l'origine e lo sviluppo di un sistema innovativo. Gli studi sui TIS hanno quindi il pregio di aver spostato l'attenzione dal 
tradizionale fallimento del mercato (market failure), che ha influenzato le politiche sulla sostenibilità degli ultimi venti anni, al fallimento del sistema (system failure). In ogni caso gli studi sui TIS non hanno invertito la logica sottostante la GT che focalizza la ricerca sul processo di formazione e diffusione delle innovazioni ambientali.

Un altro importante framework nella GT è come detto la multi-level perspective (MLP) (Geels 2002, 2004): essa spiega la transizione come il passaggio da una configurazione storica dominante (non sostenibile) a una nuova, attraverso l'interazione di processi a tre diversi livelli: nicchia (livello micro), regime socio-tecnologico (livello meso) e landscape (livello macro). Il concetto chiave è quello di "regime socio-tecnologico", una struttura stabile, caratterizzata da un insieme correlato di prodotti, tecnologie, conoscenze tecnico-scientifiche, regole di mercato, norme, regolamenti, pratiche, infrastrutture fino a comprendere la cultura e i simbolismi. Il regime socio-tecnologico conferisce orientamento e coordinamento all'attività degli agenti e quindi la sua natura intrinseca è conservativa, in quanto consente solo innovazioni incrementali e comunque nell'alveo del sentiero dominante (path dependence) e si oppone alla più ampia e profonda trasformazione strutturale imposta dalla transizione (da cui i lock in mechanisms che inibiscono il cambiamento).

Il seme del cambiamento sono le innovazioni radicali, quelle cioè in grado di accelerare fortemente la transizione verso la sostenibilità, che sono generate al livello di nicchia. Anche la MLP si concentra sulle innovazioni ambientali e quindi sulla responsabilità di primo livello di alcuni pionieri capaci di introdurre profonde innovazioni ambientali che dopo un periodo di affermazione (economica, tecnica, politica, culturale e infrastrutturale) saranno in grado di trasformare l'economia verso la sostenibilità.

Più recentemente lo stesso Geels (2014) ha riconosciuto alcune lacune della MLP nello spiegare le dinamiche della transizione verso la sostenibilità. Un primo punto debole, comune alla maggior parte degli studi sulla transizione, è il focalizzarsi sull'emergere delle innovazioni ambientali senza entrare nel dettaglio dei singoli regimi socio-tecnologici, sulle loro dinamiche interne e sugli attori che li dominano. La stabilità di un regime socio-tecnologico è spesso concettualizzata in termini di lock-in, path dependence e inerzia, senza entrare nel merito delle possibili chiusure esistenti all'emergere delle innovazioni ambientali che possono andare oltre la semplice "stabilizzazione" a arrivare fino alla sfera politica. La seconda critica, strettamente legata al primo

\footnotetext{
${ }^{1}$ In questo caso la traduzione sarebbe ambigua e quindi per evitare
} fraintendimenti si è preferito lasciare il termine in inglese. punto, è la mancanza di attenzione ai blocchi di potere economico-politico che sostengono od ostacolano lo sviluppo e l'implementazione di specifiche politiche; essi in termini Gramsciani sono definiti come "blocchi storici" e quindi sono assai contigui al suo concetto di "egemonia" quale "persistence of specific social and economic structures that systematically advantage certain groups" (Levy, Newell 2002, 87). Questo aspetto è cruciale per la velocizzazione della transizione e considera la stabilità di un regime socio-tecnologico soprattutto come una conseguenza della "resistenza" delle imprese esistenti all'introduzione del cambiamento indotto dalle innovazioni. Se quindi gli attori dominanti, e in particolare le imprese esistenti, usano il loro potere e la politica per resistere alla transizione verso la sostenibilità, è evidente l'importanza strategica della responsabilità di secondo livello, cioè del ruolo che alcuni agenti dovrebbero assumere per scardinare tali blocchi di potere e favorirla.

Tuttavia l'elemento che molti geografi hanno individuato come maggiormente critico è la mancanza di una concettualizzazione chiara sul ruolo del territorio (in inglese riferito alla parola "space") nella transizione (Coenen et al. 2012; Coenen, Truffer 2012; Hansen, Coenen 2015; Murphy 2015). Un approccio territoriale, tra l'altro, consentirebbe di spiegare la distribuzione disomogenea a livello spaziale dei risultati della transizione verso la sostenibilità e le diverse topologie di organizzazione spaziale con cui si manifesta (Strambach, Pflitsch 2020). In altre parole, alcuni fattori territoriali di fatto posizionati tra l'impresa e il mercato possono incidere fortemente sul ritmo, la portata e le diverse direzioni che la transizione verso la sostenibilità può assumere. Anche il processo di innovazione, cruciale per la responsabilità di primo livello, può avvenire in parte a livello territoriale, grazie a fattori geografici localizzati, di cui possono beneficiare tutte le imprese ivi localizzate che condividono certi valori e reti sociali (Chaminade, Randelli 2020).

Un approccio territoriale allo studio della transizione consentirebbe quindi di includere nell'analisi elementi ecologici, sociali, di governance, di salute pubblica, etc., per loro natura non confinabili al livello di singola impresa. Alcuni autori considerano l'approccio territoriale cruciale anche per le politiche (Barca et al. 2012), le cui strategie dovrebbero tenere conto delle differenze economiche, sociali, politiche e istituzionali al fine di risultare efficaci sia al livello locale che globale. Per esempio, l'approccio territoriale delle politiche è stato riconosciuto essenziale da importanti organismi internazionali (OECD, FAO e UNCDF 2016) per una rapida transizione verso un modello di cibo sostenibile.

In definitiva, un approccio territoriale alla transizione accompagna alla responsabilità di primo livello degli 
attori locali un ruolo di primo piano del territorio in cui tali attori vivono e operano. In un quadro territoriale la responsabilità di primo livello non risulta esclusivamente individuale ma può essere condivisa e partecipata con gli altri attori locali. Una responsabilità di primo livello "territoriale" ci offre una prospettiva nuova ancora non esplorata in profondità nella letteratura. Alcuni autori sostengono che la scala territoriale può fungere da mesospazio (Sonnino et al. 2016; Lamine et al. 2019), dove i processi trasformativi che avvengono al livello individuale (imprese, consumatori, loro associazioni) possono essere mediati insieme a processi trasformativi alla macro scala (ad es. cambiamenti economici e/o ambientali globali). La scala territoriale, e, in tal senso, gli spazi di governance a livello territoriale, consentono una più facile individuazione delle istituzioni e degli attori che devono essere coinvolti e quindi delle loro responsabilità, alimentando un clima di fiducia reciproca e una maggiore collaborazione e cooperazione tra gli attori al fine di una più rapida transizione verso la sostenibilità. Una responsabilità condivisa a livello spaziale che consenta di incidere sul ritmo e la portata della transizione e superare alcuni meccanismi di chiusura è un tema di discussione molto promettente per la Geografia (Frantzeskaki et al. 2018; Grillitsch, Sotarauta 2020; Binz et al. 2020; Chaminade, Randelli 2020) che potrebbe aprire scenari di ricerca e di policy nuovi.

\section{Responsabilità di secondo livello}

La responsabilità (di primo livello) impone agli agenti degli obblighi morali che, a loro volta, devono essere perseguiti attraverso azioni concrete. Il più delle volte, e nel caso della transizione verso la sostenibilità virtualmente sempre, queste azioni "riparatrici" comportano degli oneri - finanziari e non - per gli agenti di primo livello. Per quanto riguarda l'industria dei combustibili fossili, per esempio, le responsabilità (di primo livello) delle compagnie petrolifere potrebbero richiedere che esse "decarbonizzino" il proprio business; o, addirittura, che compensino il danno prodotto dagli impatti dei cambiamenti climatici in larga misura prodotti dai "loro" combustibili fossili. Per soddisfare questi obblighi le compagnie petrolifere dovrebbero modificare i loro comportamenti in modo sostanziale: a tale fine sosterrebbero, molto verosimilmente, costi notevoli, intesi in senso ampio che include anche i costi non-monetari, come per esempio i costi-opportunità. Anche se queste imprese riuscissero a trasferire parte di tali costi ad altri soggetti - per esempio, i consumatori - è estremamente improbabile che esse soddisfino i propri obblighi morali spontaneamente. Ciò, infatti, richiederebbe che l'urgenza morale delle loro responsabilità motivasse direttamente, quasi automaticamente, la loro azione. Alcune prospettive filosofiche, per esempio Gardiner (2011) e Jamieson (2014), con riferimento alle responsabilità per i cambiamenti climatici, ritengono che ciò sia possibile e sostengono che le "ragioni giustificative", cioè le responsabilità di primo livello degli agenti, rinforzano la loro motivazione a modificare le proprie azioni. Se ciò non avviene, sostengono, è perché la giustificazione delle loro responsabilità è inadeguata.

Alternativamente, e a nostro parere più realisticamente, è necessario ricorrere alla nozione introdotta sopra di responsabilità di secondo livello, cioè la responsabilità di altri agenti di assicurare che gli agenti di primo livello soddisfino le loro responsabilità (di primo livello) (O’Neill 2001, 2005; Caney 2014).

Una prima possibilità, a fronte della non soddisfazione da parte degli agenti di primo livello degli obblighi morali che derivano dalla loro responsabilità, sarebbe che altri agenti adempiessero a tali responsabilità (Shue 1996, 71-73; Caney 2005). Questa circostanza, per quanto plausibile, sembra insoddisfacente nel contesto della transizione verso la sostenibilità, in quanto sarebbe una risposta esclusivamente reattiva, ove invece sarebbe necessario muoversi proattivamente.

Una opzione più articolata, e in questo contesto decisamente più soddisfacente, arriva da Caney (2016a,b) il quale sostiene che quando alcuni agenti non soddisfano le proprie responsabilità (di primo livello), possono essere adottate quattro strategie alternative:

i. fissare obiettivi meno ambiziosi;

ii. includere fra le giustificazioni alla responsabilità considerazioni addizionali a quelle morali;

iii. condividere parte dell'onere delle azioni richieste dalla responsabilità di primo livello con altri agenti;

iv. cambiare la struttura di incentivi del contesto in cui gli agenti di primo livello operano.

$\mathrm{Nel}$ contesto della transizione verso la sostenibilità, tuttavia, le strategie i) e ii) sembrano ancora per lo più reattive; mentre la strategia iii) sembra solo moderatamente proattiva, in quanto aspira, al massimo, a modificare il comportamento degli agenti inducendoli esclusivamente a mettere in pratica azioni e comportamenti che altrimenti avrebbero evitato. In generale, quindi, le strategie da i) a iii) non sembrano adeguate a indurre gli agenti di primo livello a soddisfare le proprie responsabilità (di primo livello) in vista della transizione verso la sostenibilità.

A tale proposito è più utile, invece, la strategia prospettata al punto iv), in cui gli agenti (di primo livello) sono indotti a rispondere alle proprie responsabilità ( $\mathrm{di}$ 
primo livello) attraverso la modificazione del contesto sociale, politico, economico e legale in cui operano. Questa strategia, in altri termini, promuove il soddisfacimento delle responsabilità (di primo livello) influenzando le opportunità, i limiti e gli incentivi che gli agenti coinvolti nella transizione verso la sostenibilità hanno. Seguendo Caney (2005, 769; 2014, 134-146; 2016a, 9-10), gli elementi fondamentali per articolare questa nozione di responsabilità di secondo livello sono i "compiti" - cioè ciò che deve essere fatto per favorire la transizione verso la sostenibilità - e gli "agenti" (di secondo livello). Incrociando compiti con agenti è infatti possibile sviluppare un sistema coerente e inclusivo di responsabilità di secondo livello.

In senso lato, la giustificazione normativa alla responsabilità di secondo-livello, cioè il motivo per cui gli agenti di secondo-livello hanno un obbligo morale di intraprendere determinati compiti è che essi, così facendo, possono fare la differenza in modo sostanziale. Cioè, sulla base del principio dello zio (dell'Uomo Ragno) Ben "il potere comporta responsabilità", è possibile sostenere che gli agenti di secondo livello che hanno il potere di indurre e/o forzare gli agenti di primo livello a soddisfare le proprie responsabilità (di primo livello), hanno la responsabilità (di secondo livello) di farlo, dati il loro maggiore coinvolgimento e contiguità con i nodi e le dinamiche della transizione.

Sei sono i compiti principali che gli agenti di secondo livello hanno in relazione alla transizione verso la sostenibilità:

1. stabilire il quadro legale e politico per consentire la transizione verso la sostenibilità;

2. creare di meccanismi di enforcement che includano strumenti di trasparenza e di accountability;

3. disincentivare/incentivare comportamenti sfavorevoli/favorevoli alla transizione verso la sostenibilità;

4. promuovere la ricerca (per esempio sulle tecnologie pulite, sulle energie rinnovabili), trasferirla e supportare l'applicazione dei risultati di queste ricerche, anche nei paesi meno sviluppati;

5. diffondere norme sociali e buone pratiche che, per esempio, sostengano il riconoscimento della pericolosità dei combustibili fossili, scoraggino modelli socio-economici intensivi in termini di emissioni, e più in generale cerchino di modificare nel senso opportuno il comportamento degli agenti coinvolti nella transizione;

6. vincere le resistenze molto spesso, iniziative volte a cambiare i comportamenti dei regimi esistenti incontrano notevoli resistenze da parte degli incumbents. Per superare tali resistenze è necessario pertanto porre in essere una serie di azioni in grado di destabilizzare tali regimi.
Quali sono gli agenti di secondo livello più opportuni per realizzare i compiti sopra elencati? O, più specificamente, quali sono gli agenti di secondo livello che hanno la responsabilità (di secondo livello) di indurre gli agenti di primo livello a orientare la propria azione verso la ricerca della sostenibilità? La risposta, ovviamente, varia a seconda del compito. Alcuni "tradizionali" agenti di secondo livello - stati (governi) e organizzazioni internazionali - giocano sicuramente un ruolo centrale. Tuttavia, altri agenti meno ovvi rivestono ruoli importanti.

Per i compiti 1 e 2 - istituzioni ed enforcement - nel corrente ordine internazionale Westphaliano di sovranità statale, sembrerebbe che solo gli stati abbiano il potere di stabilire e implementare il quadro legale e politico per la transizione verso la sostenibilità. Per altri versi, tuttavia, anche i cittadini, e in senso più ampio, la società civile possono giocare un ruolo importante facendo venire meno il consenso ai governi che non dimostrano impegno sufficiente nei confronti della sostenibilità. Dato che la transizione verso la sostenibilità comporta, in tutta verosimiglianza, azioni coordinate a livello internazionale, e che quindi le strategie e le politiche nazionali dovrebbero successivamente essere coordinate e integrate, si può assumere che anche le organizzazioni internazionali - la UNFCCC, il GEF, la UE, il WTO, la World Bank, ecc. - siano agenti di secondo livello rispetto a questo compito.

Per quanto riguarda il compito 3, sono ancora gli stati ad avere un ruolo importante; ma in questo caso il ruolo delle organizzazioni internazionali è addirittura maggiore, poiché a esse spetta la definizione di coerenti strategie internazionali verso la sostenibilità attraverso accordi di collaborazione sovranazionale (Maltais 2014).

Gli altri compiti suggeriscono invece agenti di secondo livello meno convenzionali. Il compito 4 dipende largamente dall'attività di ricerca, dall'innovazione e dalla sua diffusione. In questo caso sono gli istituti nazionali di ricerca, le università, i centri di ricerca, le agenzie per l'innovazione a rivestire il ruolo centrale. Gli stati e le organizzazioni internazionali possono, al più, favorire questi attori attraverso il finanziamento della ricerca, la promozione di partenariati per la ricerca e l'innovazione, ecc.

Per quanto riguarda le norme sociali e le buone pratiche - compito 5 - gli attori principali sono ancora più inattesi: come Caney $(2014,140)$ suggerisce per la diffusione di tali norme e pratiche "... a significant role can be played by figures as diverse as church leaders, poets, novelists, charismatic individuals, and gifted communicators".

Analogamente, nel caso dell'ultimo compito - vincere le resistenze degli incumbents - gli agenti di secondo livello più efficaci risultano essere coloro che sono in grado di comunicare nel modo più efficace - e tuttavia 
oggettivo e affidabile - la necessità, i modi e i vantaggi della transizione verso la sostenibilità. Per esempio, scienziati capaci di parlare in modo semplice e comprensibile, giornalisti scientifici e altre tipologie di giornalismo investigativo, organizzazioni per la divulgazione ambientale e così via. In aggiunta a essi altri agenti cruciali per vincere le resistenze degli incumbents sono quelli che destabilizzano il loro contesto economico e finanziario, per esempio attraverso iniziative di disinvestimento.

Questo quadro di responsabilità di secondo livello ha importanti implicazioni normative e pratiche per la transizione verso la sostenibilità.

In termini normativi tale concetto di responsabilità di secondo livello è in grado di indicare azioni e strategie diverse da quelle usualmente ritenute necessarie per raggiungere la sostenibilità. Inoltre, identifica un gruppo più ampio di agenti (di secondo livello) che fanno riferimento a basi morali diverse e più cogenti a giustificazione delle proprie azioni.

In termini pratici, incrociando i compiti con gli agenti, si rende evidente che numerosi agenti di secondo livello - non solo gli stati e le organizzazioni internazionali, ma anche scienziati, giornalisti, comunità, individui carismatici il mondo dell'economia e della finanza possono in modi diversi dettare e trainare la transizione.

La individuazione puntuale di tali agenti di secondo livello è, però, possibile solo con riferimento a specifici contesti e quindi con riferimento ad analisi empiriche. $\mathrm{E}$ utile, però, individuare, nel prossimo paragrafo, il quadro di riferimento entro cui tali agenti di secondo livello - che in una prospettiva pragmatica possiamo definire "agenti di destabilizzazione (primari e operativi)" - si attivano per contribuire a vincere il potere e la resistenza degli incumbents, con l'obiettivo di favorire la transizione verso la sostenibilità.

\section{Il ruolo degli agenti di destabilizzazione nella transizione verso la sostenibilità}

Nel promuovere la transizione verso la sostenibilità gli "agenti di destabilizzazione" - come a livello più operativo appare opportuno definire quelli che nelle sezioni precedenti erano gli "agenti di secondo livello" - si scontrano inevitabilmente con la resistenza degli incumbents. Il concetto di resistenza è cruciale: Hess (2014, 279), per esempio, sottolinea che proprio nel caso della transizione verso la sostenibilità: "the political contestation by the incumbent industrial regime is so well organized that it should be at the center of the analytical framework".

Per vincere tale resistenza è quindi necessario che gli agenti di destabilizzazione si concentrino sui "pun- ti sensibili di intervento" attraverso i quali essi possono "indirizzare" e/o "spostare" (Farmer et al. 2019) un sistema socio-economico verso la sostenibilità.

Ciò è tuttavia complesso, perché molto spesso gli incumbents formano un blocco storico stabile ed egemonico orientato a mantenere lo status quo, come accennato sopra. Gli incumbents e i rispettivi governi sono tipicamente, infatti, reciprocamente dipendenti e condividono l'interesse di preservare la stabilità del business al fine di promuovere la crescita economica. I primi, dipendono dai governi che forniscono il contesto operativo generale (diritti di proprietà, regole di scambio, strutture di governance) e il sostegno pubblico sotto forma di sussidi, protezione tariffaria, agevolazioni fiscali, servizi di informazione e ricerca. Allo stesso modo, i governi e i sistemi socio-economici dipendono strettamente dalla crescita economica - almeno nei paesi capitalisti - e quindi favoriscono sistematicamente gli interessi degli incumbents, visto che questi ultimi sono funzionali a tale crescita. Newell e Paterson (1998) sostengono, per esempio, che le grandi imprese incumbent hanno un "potere strutturale", in quanto i governi dipendono da esse per fornire posti di lavoro, tasse, ricchezza e dinamismo sociale.

Quindi, per poter comprendere come gli agenti di destabilizzazione possano operare, è necessario analizzare le forme di potere che gli incumbents, coerentemente con l'analisi Gramsciana sulle caratteristiche dell'egemonia, utilizzano per resistere alle spinte esogene al cambiamento, segnatamente nel nostro caso a quello connesso alla transizione verso la sostenibilità

In primo luogo, gli incumbents utilizzano forme strumentali di potere - denaro, autorità, accesso ai media, capacità di lobbying e reti - per perseguire i loro interessi e raggiungere i loro obiettivi, ivi compreso il mantenimento dello status quo. Secondariamente, essi utilizzano forme di potere discorsivo, attraverso il quale plasmano, manipolano e orientano l'opinione pubblica in loro favore. Una terza forma di potere è quella istituzionale. Essa è radicata nelle culture politiche, nell'ideologia e nelle strutture di governance, e sostanzialmente consente agli incumbents di dettare la propria agenda ai politici. La quarta forma di potere è materiale e riguarda le iniziative che gli incumbents, attingendo alle loro capacità manageriali, tecniche e finanziarie, mettono in campo per rendere meno controverse le implicazioni delle loro tecnologie e attività.

Ovviamente queste forme di potere nella realtà non sono separate l'una dall'altra, anzi sono interdipendenti e tendono a rinforzarsi reciprocamente. Un esempio, fra i tanti, dell'uso congiunto di potere materiale e discorsivo nell'industria petroliofera riguarda l'enfasi posta dagli incumbents sulle innumerevoli innovazioni tecno- 
logiche a basso impatto ambientale - per esempio dispositivi di desolforazione dei gas di scarico, tecnologie di polverizzazione del carbone, gassificazione del carbone - che, secondo l'industria stessa, avrebbero contribuito alla nascita del "carbone pulito"; o sulle tecniche di cattura e stoccaggio del carbonio in atmosfera (CCS), che nonostante la loro fattibilità tecnica e il loro potenziale, presentano ancora notevoli incertezze in termini di scala necessaria e fattibilità commerciale.

In questa prospettiva gli agenti di destabilizzazione hanno specificità e capacità diverse e quindi svolgono ruoli differenti rispetto alle varie forme di potere degli incumbents. Di seguito viene brevemente delineato quali agenti di destabilizzazione sono più adatti ad affrontare le diverse forme di potere degli incumbnents in vista di favorire la transizione verso la sostenibilità.

A tale fine è opportuno sottolineare che per erodere il potere e la resistenza degli incumbents è necessario innanzitutto modificare i contesti sociali, economici e politici in cui essi operano. Solo successivamente, infatti possono intervenire gli agenti di destabilizzazione coinvolti in compiti più operativi come la disincentivazione finanziaria e la ricerca di nuove teologie. Ovviamente a livello del framework proposto qui è impossibile specificare quali sono le specificità e le leve di destabilizzazione entro questi contesti. Tali analisi devono avvenire con specifico riferimento al contesto in analisi: per esempio un'analisi di questo tipo è stata condotta con riferimento all'industria petrolifera (Grasso 2022).

Per ragioni di chiarezza, gli agenti coinvolti nelle attività del primo tipo possono essere considerati forze "primarie"; quelli che utilizzano i mercati e le normative per guidare/modificare il comportamento degli incumbents sono definiti forze "operative". Sebbene la distinzione tra agenti primari e operativi, ancora una volta, sia sfumata nella realtà in quanto entrambi possono focalizzarsi sulle stesse azioni, si assume che, per quanto riguarda la transizione verso la sostenibilità, gli agenti primari di destabilizzazione siano quelli che mirano a "spostare" la dinamica del sistema socio-economico modificandone sostanzialmente le regole e le traiettorie (ad esempio i concetti chiave, i valori e le istituzioni); gli agenti operativi invece quelli che "indirizzano" il sistema socio-economico per spingerlo su nuove traiettorie senza tuttavia modificarne le dinamiche sottostanti (Farmer et al. 2019). In generale, gli agenti primari di destabilizzazione pongono le basi in un modo bottom-up e quasi anarchico per preparare le società a riconoscere e accettare la necessità della transizione verso la sostenibilità e delle sue implicazioni. Su questo terreno "fertilizzato", gli agenti operativi di destabilizzazione possono/devono introdurre le misure effettivamente volte a indurre concretamente gli incumbents al cambiamento.
Alla luce di queste considerazioni e con riferimento agli agenti di destabilizzazione sembra che - sempre con riferimento alla transizione verso la sostenibilità - quelli primari - per esempio, individui carismatici e movimenti sociali - siano i più indicati per affrontare le forme strumentali, discorsive e istituzionali del potere degli incumbents, mentre quelli operativi - per esempio, il mondo della ricerca, le aziende che introducono cambiamenti radicali (per esempio molte start-ups) e gli attori finanziari - paiono le forze più efficaci per erodere il loro potere materiale.

In particolare, per quanto riguarda il primo gruppo di forme di potere (strumentale, discorsivo, istituzionale), gli individui carismatici hanno un ruolo di primo piano nel sensibilizzare le società e le relative dinamiche culturali alle sfide poste dalla corrente crisi ecologica globale. Si pensi alla straordinaria capacità dei "leader carismatici” (ad esempio, Papa Francesco o l'attivista svedese Greta Thunberg) per quanto riguarda specificamente la loro capacità di mobilitare rapidamente la società civile. Il loro ruolo distintivo è quello di aggregare e catalizzare le pressioni che provengono dalla società civile e di trasformarle in una lenta ma costante "ondata di forze nuove" per sfidare il potere degli incumbents, soprattutto su basi strumentali, discorsive e istituzionali.

Tale "ondata di forze nuove" sono solitamente i cosiddetti "movimenti sociali", cioè reti di interazioni informali tra una pluralità di individui, gruppi e/o organizzazioni, impegnati in conflitti politici o culturali, sulla base di identità collettive condivise (Diani 1992). In termini pratici, i movimenti sociali sono coalizioni di individui e organizzazioni della società civile e del settore pubblico e privato. L'azione collettiva dei movimenti sociali è un fattore chiave nei cambiamenti dei sistemi umani, sociali ed economici (Della Porta, Diani 2006, 33-63). Per quanto riguarda la transizione verso la sostenibilità, i movimenti sociali possono essere considerati altri fondamentali agenti primari di destabilizzazione. Molto spesso essi sono riusciti ad affermarsi come forze contro-egemoniche attraverso lo sviluppo di un'adeguata rappresentazione della crisi ecologica globale come minaccia per l'umanità. Attraverso questa narrativa le forza contro-egemoniche rappresentate dai movimenti sociali cerca di erodere i poteri strumentali, discorsivi e istituzionali degli incumbents che si oppongono alla transizione verso la sostenibilità.

Per quanto riguarda il potere materiale degli incumbents che si oppone alla transizione verso la sostenibilità, gli agenti operativi di destabilizzazione hanno invece un ruolo prioritario. Fra questi il mondo della ricerca e i nuovi imprenditori che emergono a livello di nicchia svolgono l'azione fondamentale di stimolare l'innovazione tecnologica e sociale. In questo caso il terreno per erodere il pote- 
re degli incumbents risiede principalmente nello sviluppo di nuovi prodotti, servizi e modelli di business, nel contribuire alla creazione di un mercato per le nuove tecnologie e nel diffondere tali tecnologie. A un livello diverso, il mondo della ricerca dovrebbe anche cercare di plasmare le narrative sociali e l'inquadramento dei problemi, di fare lobby per politiche e normative specifiche, di sviluppare standard industriali, e plasmare le aspettative collettive (Binz et al. 2016; Rosenbloom et al. 2016).

Altri agenti operativi di destabilizzazione importanti nell'ambito della transizione verso la sostenibilità sono, come anticipato, le istituzioni finanziarie: per lo più fondi pensione e fondi sovrani e banche centrali, di sviluppo e commerciali. Per erodere il potere materiale degli incumbents, le istituzioni finanziarie dovrebbero prima di tutto cessare di finanziare le attività legate a business non più sostenibili come quello dei combustibili fossili.

\section{Conclusioni}

Il framework teorico delineato, per quanto "a maglie larghe", è utile in termini analitici e, come detto, ha l'ambizione di fornire le basi per future analisi contestualizzate della transizione verso la sostenibilità. Ovviamente, la realtà è molto meno netta e univoca: per esempio, alcune forme di potere si rafforzano a vicenda e quindi la loro erosione richiede la pressione concertata di molteplici agenti di destabilizzazione, primari e operativi: segnatamente i poteri materiali e discorsivi spesso si autoalimentano e quindi sia i movimenti sociali che gli attori finanziari sono decisivi nell'affrontare queste forme di potere.

In una prospettiva complementare lo spostamento dei confini della responsabilità di primo livello in modo esclusivo dagli agenti (imprese, consumatori, associazioni) al territorio è un'evoluzione in atto che seppur ancora non ben definita, sembra essere promettente. Nel campo del cibo, per esempio, questo tipo di responsabilità territoriale condivisa (Sonnino et al. 2016; Lamine et al. 2019) viene vista come una modalità per accelerare la transizione verso la sostenibilità superando i limiti di un approccio "individuale" alle responsabilità che di fatto inibisce la fiducia reciproca e la cooperazione. Esperienze internazionali come i Food Policy Councils o italiane come i Biodistretti e le Politiche per il Cibo di alcune città italiane - tra cui Torino, Milano, Livorno e recentemente Roma - consentono di mettere intorno allo stesso tavolo amministratori locali, produttori, associazioni di consumatori e quindi chiarire le responsabilità individuali e quelle eventualmente reciproche.

Il ruolo del territorio come mediatore di interessi diversi degli attori locali è di forte interesse per la Geo- grafia (Hansen, Coenen 2015; Binz et al, 2020; Grillitsch, Sotarauta 2020) e i risultati delle analisi potrebbero portare linfa nuova agli studi sulla transizione verso la sostenibilità. Infatti, in altri contesti, come per esempio il settore energetico, i blocchi di potere sono di tali dimensioni che una semplice azione alla scala territoriale locale non sarebbe in grado di stimolare la presa di responsabilità dei soggetti coinvolti. In un contesto simile risulta pertanto cruciale la responsabilità di secondo livello alle diverse scale, anche locale, ma soprattutto nazionale e globale, al fine di superare i meccanismi di chiusura ed accelerare la transizione verso la sostenibilità.

Il framework prospettato in questo lavoro non è quindi un punto di arrivo ma una proposta operativa per includere nell'analisi della transizione verso la sostenibilità più elementi, quali il potere, la politica, gli agenti di destabilizzazione, il ruolo del territorio, dalle cui strategie dipende molto del futuro dell'umanità su questo pianeta. Le ricerche future devono chiarire con maggiore specificità le singole responsabilità anche con lo scopo di proporre nuove modalità di governance locale e globale, capaci di incidere sia sul ritmo che sulla portata della transizione. Tuttavia, riteniamo, che il framework proposto renda possibile mappare il "chi-fa-cosa", per così dire, nella transizione verso la sostenibilità e che quindi possa essere uno riferimento teorico cruciale per le analisi empiriche di specifici incumbents e/o contesti volte alla comprensione del potenziale degli agenti di destabilizzazione per favorire la transizione verso la sostenibilità.

\section{Riferimenti bibliografici}

Bergek, A., Jacobsson, S., Carlsson, B., Lindmark,, S., Rickne, A. (2008). Analyzing the functional dynamics of technological innovation systems: A scheme of analysis. Research Policy, 37, 407-429. DOI: 10.1016/j. respol.2007.12.003

Binz, C., Coenen, L., Murphy, J. T. (2020). Geographies of transition-From topical concerns to theoretical engagement: A commentary on the transitions research agenda. Environmental Innovation and Societal Transitions, 34, 1-3. DOI: 10.1016/j.eist.2019.11.002

Binz, C., Harris-Lovett, S., Kiparsky, M., Sedlak, D. L., Truffer, B., (2016). The thorny road to technology legitimation - Institutional work for potable water reuse in California. Technological Forecasting and Social Change, 103, 249-263. DOI: 10.1016/j.techfore.2015.10.005

Caney, S. (2005). Cosmopolitan justice, responsibility, and global climate change. Leiden Journal of International 
Law, 18, 747-775. DOI: 10.1017/S0922156505002992

Caney, S. (2014). Two kinds of climate justice: Avoiding harm and sharing burdens. The Journal of Political Philosophy, 22 (2), 125-149. DOI: 10.1111/jopp.12030

Caney, S. (2016a). Climate change and non-ideal theory: Six ways of responding to noncompliance. In Heyward, C. e Roser, D. (Eds.), Climate justice in a non-ideal world. Oxford, Oxford University Press, 21-42.

Caney, S. (2016b). The struggle for climate justice in a non-ideal world. Midwest Studies In Philosophy, 40 (1), 9-26. DOI: $10.1111 / \mathrm{misp} .12044$

Carlsson, B., Stankiewicz, R. (1991). On the nature, function and composition of technological systems. Evolutionary Economics, 1, 93-118.

Chaminade, C., Randelli, F. (2020). The role of territorially embedded innovation ecosystems accelerating sustainability transformations: A case study of the transformation to organic wine production in Tuscany (Italy). Sustainability, 12 (11), 4621. DOI: 10.3390/su12114621

Coenen, L., Truffer B. (2012). Places and spaces of sustainability transitions: Geographical contributions to an emerging research and policy field. European Planning Studies, 20 (3), 367-374. DOI: 10.1080/09654313.2012.651802

Coenen, L., Benneworth, P., Truffer, B. (2012). Towards a spatial perspective on sustainability transitions. Research Policy, 41, 968-979. DOI: 10.1016/j.respol.2012.02.014

Della Porta, D., Diani, M. (2009). Social movements: An introduction (Second Edition), Oxford, Blackwell Publishing.

Diani, M. (1992). The concept of social movement. The Sociological Review, 40 (1), 1-25.

Farmer, J. D., Hepburn, C., Ives, M. C., Hale, T., Wetzer, T., Mealy, P., Way, R., (2019). Sensitive intervention points in the post-carbon transition. Science, 364 (6436), 132-134. DOI: $10.1126 /$ science.aaw7287

Frantzeskaki, N., van Steenbergen, F., Stedman, R. C. (2018). Sense of place and experimentation in urban sustainability transitions: the Resilience Lab in Carnisse, Rotterdam, The Netherlands. Sustainability Science, 13 (4), 1045-1059. DOI: 10.1007/s11625-018-0562-5

Gardiner, S. M., (2011). Is no one responsible for global environmental tragedy? Climate change as a challenge to our ethical concepts. In Arnold, D. (Ed.), The ethics of global climate change. Cambridge, Cambridge University Press, 38-59.

Gardiner, S. M., (2017). Climate ethics in a dark and dangerous time. Ethics, 127, 430-465. DOI: 10.1086/688746
Geels, F. W. (2002). Technological transitions as evolutionary reconfiguration processes: a multi-level perspective and a case-study. Research Policy, 31, 1257-1274. DOI: 10.1016/S0048-7333(02)00062-8

Geels, F. W. (2004). From sectoral systems of innovation to sociotechnical systems: insights about dynamics and change from sociology and institutional theory. Research Policy, 33, 897-920. DOI: 10.1016/j.respol.2004.01.015

Geels, F. W. (2014). Reconceptualising the co-evolution of firms-in-industries and their environments: Developing an inter-disciplinary Triple Embeddedness Framework. Research Policy, 43, 261-277. DOI: 10.1016/j. respol.2013.10.006

Grabher, G., Ibert, O., Flohr, S. (2008). The neglected king: The customer in the new knowledge ecology of innovation. Economic Geography, 253-280. DOI: 10.1111/ j.1944-8287.2008.tb00365.x

Grasso, M. (2007). A normative ethical framework in climate change. Climatic Change, 81(3), 223-246. DOI: 10.1007/s10584-006-9158-7

Grasso, M. (2022) From Big Oil to Big Green. Holding the oil industry to account for the climate crisis. Cambridge, Ma. and London, MIT Press.

Grasso, M., Markowitz, E. M. (2015). The moral complexity of climate change and the need for a multidisciplinary perspective on climate ethics. Climatic Change, 130 (3), 327-334. DOI: 10.1007/s10584-014-1323-9

Grasso, M., Tàbara, J. D. (2019). Towards a moral compass to guide sustainability transformations in a HighEnd Climate Change world. Sustainability, 11, 2971. DOI: $10.3390 /$ su1110297

Graziano, P. R., Forno, F. (2012). Political consumerism and new forms of political participation: The Gruppi di Acquisto Solidale in Italy. The Annals of the American Academy of Political and Social Science, 644 (1), 121-133. DOI: $10.1177 / 0002716212454839$

Grillitsch, M., Sotarauta, M. (2019). Trinity of change agency, regional development paths and opportunity spaces. Progress in Human Geography, 44 (4), 704-723. DOI: 10.1177/0309132519853870

Hansen T., Coenen L. (2015). The geography of sustainability transitions: Review, synthesis and reflections on an emergent research field. Environmental Innovation and Societal Transitions, 17, 92-109. DOI: 10.1016/j.eist.2014.11.001

Hess, D. J. (2014). Sustainability transitions: A political coalition perspective. Research Policy, 43, 278-283. DOI: 10.1016/j.respol.2013.10.008 
Hölscher, K., Wittmayer, J. M. e Loorbach, D. (2018). Transition versus transformation: What's the difference? Environmental Innovation and Societal Transitions, 27, 1-3. DOI: $10.1016 /$ j.eist.2017.10.007

Holt-Giménez, E., Altieri, M. A. (2013). Agroecology, food sovereignty, and the new green revolution. Agroecology and Sustainable Food Systems, 37 (1), 90-102. DOI: 10.1080/10440046.2012.716388

IPBES (2019). Global assessment report on biodiversity and ecosystem services of the Intergovernmental SciencePolicy Platform on Biodiversity and Ecosystem Services, E. S. Brondizio, J. Settele, S. Díaz, and H. T. Ngo (editors). Bonn, Germany, IPBES Secretariat.

IPCC (2021) Climate Change 2021: The Physical Science Basis. Contribution of Working Group I to the Sixth Assessment Report of the Intergovernmental Panel on Climate Change, Cambridge, Cambridge University Press.

Jamieson, D. (2014). Reason in a dark time: Why the struggle against climate change failed--And what it means for our future, New York, Oxford University Press.

Kivimaa, P., Laakso, S., Lonkila, A. e Kaljonen, M. (2021). Moving beyond disruptive innovation: A review of disruption in sustainability transitions. Environmental Innovation and Societal Transitions, 38, 110-126. DOI: 10.1016/j.eist.2020.12.001

Lamine, C., Magda, D., Amiot, M. J. (2019). Crossing sociological, ecological, and nutritional perspectives on agrifood systems transitions: Towards a transdisciplinary territorial approach. Sustainability, 11 (5), 1284.

Levy, D. L., Newell, P. J. (2002). Business strategy and international environmental governance: Toward a NeoGramscian synthesis. Global Environmental Politics, 2 (4), 84-101. DOI: 10.1162/152638002320980632

Lundvall, B. A., (ed) (1992). National system of innovation: Towards a theory of innovation and interactive learning, London, Pinter Publishers.

Maltais, A. (2014). Failing international climate politics and the fairness of going first. Political Studies, 62 (3), 618-633. DOI: 10.1111/1467-9248.12073

Markard, J., Truffer, B. (2008). Technological innovation systems and the multi-level perspective: towards an integrated framework. Research Policy, 37, 596-615. DOI: 10.1016/j.respol.2008.01.004

Markard, J., Raven, B., Truffer, B. (2012). Sustainability transitions: An emerging field of research and its prospects. Research Policy, 41, 955-967. DOI: 10.1016/j. respol.2012.02.013.
Millennium Ecosystem Assessment (2005). Island Press (2nd edition).

Murphy, J. T. (2015). Human geography and socio-technical transition studies: Promising intersections. Environmental Innovation and Societal Transitions, 17, 73-91. DOI: 10.1016/j.eist.2015.03.002

Nelson, R. R. (ed) (1993). National innovation systems: A comparative analysis, Oxford, Oxford University Press.

Newell, P., Paterson, M. (1998). A climate for business: global warming, the state and capital. Review of International Political Economy, 5 (4), 679-703.

O’Neill, O. (2001). Agents of justice. Metaphilosophy, 32 (1-2), 180-195.

O'Neill, O. (2005). The dark side of human rights. International Affairs, 81 (2), 427-439.

OECD; FAO; UNCDF (2016). Adopting a territorial approach to food security and nutrition policy, Paris, France, OECD Publishing.

Randelli, F., Rocchi, B. (2017). Analysing the role of consumers within technological innovation systems: The case of alternative food networks. Environmental Innovation and Societal Transitions, 25, 94-106. DOI: 10.1016/j. eist.2017.01.001

Rosenbloom, D., Berton, H., Meadowcroft, J. (2016). Framing the sun: A discursive approach to understanding multi-dimensional interactions within socio-technical transitions through the case of solar electricity in Ontario, Canada. Research Policy, 45, 1275-1290. DOI: 10.1016/j.respol.2016.03.012

Schot, J., Kanger, L. (2018). Deep transitions: Emergence, acceleration, stabilization and directionality. Research Policy, 47, 1045-1059. DOI: 10.1016/j.respol.2018.03.009

Shue, H. (1996). Basic rights: Subsistence, affluence, and US foreign policy, Princeton, NJ: Princeton University Press.

Shue, H. (2017). Responsible for what? Carbon producer $\mathrm{CO}_{2}$ contributions and the energy transition. Climatic Change, 144 (4), 591-596. DOI: 10.1007/s10584-017-2042-9

Sonnino, R., Marsden, T. e Moragues-Faus, A. (2016). Relationalities and convergences in food security narratives: towards a place-based approach. Transactions of the Institute of British Geographers, 41 (4), 477-489. DOI: 10.1111/ tran.12137

Strambach, S., Pflitsch, G. (2020). Transition topology: Capturing institutional dynamics in regional development paths to sustainability. Research Policy, 49(7), 104006. DOI: 10.1016/j.respol.2020.104006 